\title{
Acute airway obstruction by laryngeal haematomas in acute immune thrombocytopenic purpura
}

\author{
TC Aiken, RC Collin
}

\begin{abstract}
Summary
We report the case of a 79-year-old woman with acute immune thrombocytopenic purpura who spontaneously developed laryngeal haematomas which produced acute upper airway obstruction
\end{abstract}

Keywords: immune thrombocytopenic purpura, laryngeal obstruction, ovarian carcinoma

A 79-year-old woman presented with a twoday history of a swollen tongue, bleeding from her mouth and bruising of her left arm. She was found to have a thrombocytopenia of $72 \times 10^{9} /$ 1. There was no history of recent illness. She had had uncomplicated essential hypertension and osteoarthritis for several years which her general practitioner had been treating with bendrofluazide $2.5 \mathrm{mg}$ daily and ibruprofen $400 \mathrm{mg} 8$ hourly. Both drugs were stopped on admission. On examination there were widespread purpura of the skin and sub-mucosal haematomas within the mouth. Her blood pressure and pulse were normal and she had no hepato-splenomegaly. Her haemoglobin, white cell count, clotting screen, urea and electrolytes, blood glucose and liver function were normal.

A bone marrow aspirate showed a particulate and hypercellular sample with increased numbers of morphologically normal megakaryocytes.

Erythropoietic and myelopoietic cell lines were normal with normal iron stores. A diagnosis of acute immune thrombocytopenic purpura was therefore confirmed and she was started on intravenous gammaglobulin $(0.8 \mathrm{~g} /$ kg) daily. Sixteen hours after admission she developed sudden, severe stridor. Her haemoglobin and platelet count had both dropped to $9.2 \mathrm{~g} / \mathrm{dl}$ and $<10 \times 10^{9} / 1$, respectively. A repeat clotting screen was normal and there was no evidence of haemolysis. A consultant otolaryngologist inserted a temporary tracheostomy with a pre-operative, 12-unit platelet transfusion. The tracheostomy achieved immediate relief. His findings on direct laryngoscopy were of petechial haemorrhages on the epiglottis, sub-mucosal haemorrhages of the posterior pharyngeal wall extending into the cricopharyngeal area and bilateral subepiglottic haematomas, originating from the false cords. The true cords were not visible.
She was transferred to the intensive therapy unit, was allowed to ventilate spontaneously and was given four units of red cell concenrate. She was continued on $0.8 \mathrm{~g} / \mathrm{kg} /$ day of intravenous gammaglobulin for the next four days and was given six units of platelets daily for the next five days. Two days after admission her platelet count had only marginally improved to $15 \times 10^{9} / 1$. She was therefore also given an intravenous infusion of vincristine $1.4 \mathrm{mg} / \mathrm{m}^{2}$ over four hours. Subsequently she developed a lobar pneumonia and was treated with intravenous augmentin $600 \mathrm{mg} 8$ hourly.

Six days after admission her platelet count had improved to $35 \times 10^{9} / 1$. The gammaglobulin and platelet transfusions were now stopped and replaced with an intravenous infusion of methyl-prednisolone $1.5 \mathrm{~g}$ daily for two days. Three days later her platelet count had improved to $65 \times 10^{9} / 1$ and she was given a further intravenous infusion of vincristine.

Nine days after admission, fibre-optic laryngoscopy showed complete resolution of the laryngeal haematomas and fully mobile vocal cords. The tracheostomy was removed. Unfortunately her general condition deteriorated and intravenous imipenem $1 \mathrm{~g}$ 12-hourly was commenced. The haemoglobin and white cell count remained normal with a platelet count of $71 \times 10^{9} / 1$. She continued to deteriorate and subsequently died 17 days after admission. The post mortem confirmed an infective pneumonitis and demonstrated a normal liver, spleen and gastrointestinal tract. The remainder of the post mortem was normal apart from the unexpected finding of an $11-\mathrm{cm}$ ovarian carcinoma.

\section{Discussion}

Immune thrombocytopenic purpura was first described by Werlhof in 1735 as 'morbus haemorrhagicus maculosis'. It was not until the middle of this century that its immune mechanism was deduced and the first case of a drug-induced immune thrombocytopenic purpura described.

Despite surviving this rare complication of acute immune thrombocytopenic purpura it seemed that a pneumonia was responsible for the patient's death. There were several possible causes of the immune thrombocytopenic purpura. Thiazide diuretics ${ }^{1}$ and less commonly nonsteriodal anti-inflammatory drugs ${ }^{2}$ have 


\begin{tabular}{|l|}
\hline Immune thrombocytopenic \\
purpura: causes \\
\hline - idiopathic \\
- secondary to systemic disorder: systemic \\
lupus erythematosus, chronic lymphatic \\
leukaemia, lymphoma, hyperthyroidism \\
- drug-induced \\
post-transfusion \\
\hline
\end{tabular}

\begin{tabular}{|l|}
\hline Immune thrombocytopenic \\
purpura: clinical presentation \\
\hline - skin purpura \\
- haemorrhagic mucosal bullae \\
- epistaxis \\
- gaematuria \\
- introcrantestinal haemorrhage \\
\hline
\end{tabular}

\begin{tabular}{|l|}
\hline Immune thrombocytopenic \\
purpura: treatment options \\
\hline - corticosteroids \\
- gammaglobulin \\
- vinca alkaloids \\
- planectomy \\
- plasma exchange \\
\hline
\end{tabular}

both been previously implicated. The development of hypersensitivity is unpredictable and may take hours or years. The occult ovarian carcinoma may also be postulated as a cause although, until recently, this association had not been described. Two cases report the association of immune thrombocytopenic purpura and ovarian carcinoma. One showed resolution of immune thrombocytopenic purpura with ablation of an ovarian carcinoma ${ }^{3}$ and the other reported the recurrence of an ovarian carcinoma presenting as an immune thrombocytopenic purpura. ${ }^{4}$

Immune thrombocytopenic purpura is associated with a greater proportion of young, functionally superior platelets in circulation than in thrombocytopenias of a corresponding level due to bone marrow suppression. ${ }^{5}$ Provided the platelet count is above $20 \times 10^{9} / 1$ and

1 Eisner EV, Cromwell EB. Hydrochlorthiazide dependen thrombocytopenia due to IgM antibody. $\mathcal{F} A M A 1971 ; 215$ 480

2 Meyr T, Hermann C, Wiegand V, Mathias B, Kiefel V Mueller-Eckhardt C. Immune thrombocytopenia associated with a haemorrhagic diathesis due to Ibuprofen administra tion. Clin Invest 1993; 71: 413-5.

3 Belluchi S, Dosquet C, Boval B, Wautier JL, Caen J. Association of auto-immune thrombocytopenic purpura, Grave's disease and ovarian carcinoma. Nouv Rev Fr Haematol 1991; 33: 307-9.

4 Tarraza HM, Carroll R, De Cain M, Jones M. Recurrent ovarian carcinoma: presentation as ITP and a splenic mass. Eur f Gynaecol Oncol 1991; 12: 439-43.
Learning points

- hospital admission if thrombocytopenia and haemorrhage co-exist

- if possible, stop all drugs, especially those affecting platelet function

- acute stridor is an emergency but readily correctable

- vigilance for complications in the elderly with acute immune thrombocytopenic purpura

- possible association of immune

thrombocytopenic purpura and ovarian carcinoma

there is not a concomitant clotting abnormality, serious haemorrhage is exceptional. ${ }^{5}$

Mortality due to drug-induced immune thrombocytopenic purpura is probably $<5 \%$ but this may be higher in the elderly. ${ }^{5}$ Also, haemorrhagic complications in the elderly at equivalent platelet counts appear to be more frequent. ${ }^{6}$ Treatment options for acute immune thrombocytopenic purpura with haemorrhagic complications include corticosteroids, intravenous gammaglobulin, vincristine infusions and platelet transfusions. Corticosteroids are known to be of benefit in immune thrombocytopenic purpura by acting on the vascular endothelium to improve haemostasis as well as on the reticuloendothelial mononuclear macrophage system to decrease platelet destruction. ${ }^{\text {? }}$

Intravenous gammaglobulin is thought to act on the mononuclear macrophage system and also produces immune modulation ${ }^{8}$ but its use in situations other than life-threatening emergencies is controversial. Vinca alkaloids are also thought to act on the mononuclear macrophage system by inhibiting phagocytosis of platelets. ${ }^{9}$ Slow infusions are more effective than bolus injections and vincristine is favoured because it is less myelotoxic and it stimulates thrombopoiesis. The survival time of platelet transfusions limits their benefit in immune thrombocytopenic purpura but they are recommended to achieve haemostasis in life-threatening haemorrhage. ${ }^{10}$

This case demonstrates a significant but rare complication of immune thrombocytopenic purpura which is potentially readily treatable with appropriate intervention.

5 Hardisty RM, Wetherall DJ. Blood and its disorders, 2nd edn. Oxford: Blackwell Publications, 1982; ch 25.

6 Cortelazzo S, Finnazi G, Buelli M, Moltem A, Viero P Barbui $T$. High risk of severe bleeding in aged patients with chronic ITP. Blood 1991; 77: 31-3.

7 Werkentin T, Kelton J. Current concepts in the treatment of ITP. Drugs 1990; 40: 531-42.

8 Newland AC. The use and mechanisms of actions of intravenous immunoglobulin: an update. $\mathrm{Br} \mathcal{f}$ Haematol 1989; 72: 301-5.

9 Monoharam A. Treatment of refractory ITP in adults: clinical annotations. Br f Haematol 1991; 79: 143-7.

10 British Society for Haematology. Guidelines for platelet transfusions. Transfus Med 1992; 3: 311-8. 